\title{
Plasma Formation In a Pseudospark Discharge
}

\author{
PETER CHOI, HERNAN CHUAQUi, J. LUNNEY, R. REICHLE, A. J. DAVIES, AND K. MITTAG
}

\begin{abstract}
Unique features associated with the hollow cathode recess in a pseudospark discharge have been identified from streak camera observations. A plasma is observed to form in a highly localized region immediately behind the cathode aperture prior to gas breakdown in the main discharge volume. The point-like plasma expands from an initial diameter of below 0.3 to $3 \mathrm{~mm}$, the size of the cathode aperture, in about $20 \mathrm{~ns}$, at which time the main discharge is formed starting from the axis. Numerical modeling based on swarm parameters has been carried out to investigate the plasma formation in the hollow cathode region. Results show that a combination of varying ionization rates due to the field geometry and differing mobilities of electrons and ions leads to the formation of a highly localized space-charge field on axis behind the hollow cathode. This space-charge field in turn brings about the rapid formation of a highly localized, high density of charge carriers behind the cathode aperture. It is suggested that this point-like formation of a plasma source is the phenomenon observed in the streak camera observation of the hollow cathode region.
\end{abstract}

\section{INTRODUCTION}

$\mathrm{T}$ HE pseudospark discharge is a pulsed hollow cathode-type discharge at low pressure with a constricted cathode opening and axially symmetric parallel electrodes [1], [2]. Although spark discharges at low pressure have been investigated in the past [3], [4], the physics behind the initial plasma formation leading to breakdown is not well understood. This is particularly so in a constricted hollow cathode geometry where a strongly divergent field exists inside the hollow cathode. Earlier results [5] suggest that a very small voltage on a control electrode behind the hollow cathode opening would significantly modify the breakdown characteristics of the main discharge. In addition, high intensity, well-collimated beams of electrons are observed from these discharges [2], [5], and the mechanism of the production of the beams remains unknown. Here we report a series of experiments and numerical simulation aimed at studying the initial plasma formation within the hollow cathode region, and the resulting onset of gas breakdown in the main volume.

Manuscript received October 26, 1988; revised February 1, 1989. This work was supported by the CEC under Stimulation Action Contract ST2J0198. The experimental work was performed at Imperial College, London, UK, and the numerical work was carried out at the Kernforschungszentrum Karlsruhe GmbH, FRG.

P. Choi is with the Blackett Laboratory, Imperial College, London SW7 2BZ, UK.

H. Chuaqui is on leave from the Pontificia Universidad Catolica de Chile, Santiago 22, Chile. He is now with the Blackett Laboratory, Imperial College, London SW7 2BZ, UK.

J. Lunney is with Trinity College, Dublin, Ireland.

R. Reichle is with the Universität Erlangen-Nürnberg, Erlangen, FRG

A. J. Davies is with University College, Swansea, UK

K. Mittag is with Kernforschungszentrum Karlsruhe GmbH, INR, Postfach 3640, D-7500 Karlsruhe, FRG

IEEE Log Number 8930363.

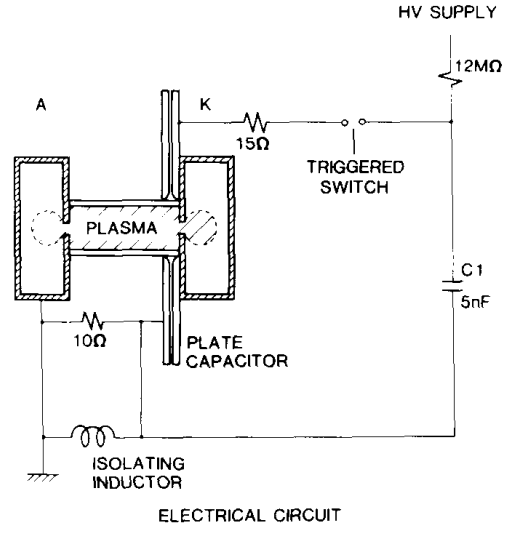

Fig. 1. Schematic of the experimental apparatus and electrical circuit.

\section{EXPERIMENTAL APParatus}

A fast-pulse system was used to study the plasma phenomena leading to the breakdown. A schematic of the experimental apparatus is shown in Fig. 1, together with the electrical circuit. The discharge is initiated in a $2-\mathrm{cm}$ diam, $8-\mathrm{cm}$ length glass tube. Both the anode and cathode are hollow, with diagnostics ports for the unrestricted observation of events taking place at the back of the electrodes. A $5-\mathrm{nF}$ primary capacitor, $\mathrm{C} 1$, is charged to the working voltage through a $12-\mathrm{M} \Omega$ current limiting resistor. The low inductance plate capacitor, which is located on one of the electrodes and forms an integral part of the discharge chamber, is pulse-charged by the $\mathrm{C} 1$ through a triggered switch. With a $15-\Omega$ damping resistor, this arrangement produces a voltage step between the anode and cathode having a rise time of under $50 \mathrm{~ns}$ and a decay time constant of over $100 \mu \mathrm{s}$, determined primarily by a $6-\mathrm{k} \Omega$ resistive voltage monitor across the plate capacitor. When the gas breaks down and the main discharge is formed, a current with a typical rise time of $12 \mathrm{~ns}$ begins to flow (Fig. 2). A $10-\Omega$ resistor is used to provide near-critical damping and thus a nonoscillatory discharge current. The resistor limits the peak current to about $2 \mathrm{kA}$ at a $32 \mathrm{kV}$ charging. This close coupling arrangement of the energy supply circuit was chosen to decrease the pulse rise time and to increase the rate of energy input at the discharge formation. This removes ambiguities in interpreting the finite time of discharge formation and the resulting finite voltage fall time.

\section{EXPERIMENTAL RESUlts}

To study the discharge formation processes in the pseudospark, high-speed streak photography in the visible re- 


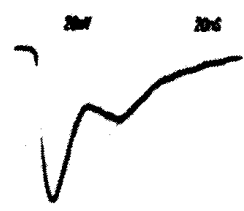

Fig. 2. Typical discharge current flowing in the plasma after voltage breakdown. The horizontal scale is $40 \mathrm{~ns} / \mathrm{div}$.

gion has been carried out using a Hadland 675 streak camera with an image intensifier attachment. The streak measurements were carried out with the viewing slit in both radial and axial directions, to look inside the hollow cathode region and at different positions in the main discharge volume. The results in the following are obtained for discharges in nitrogen at 10 mtorr pressure at a repetition rate of $1 \mathrm{~Hz}$ and a charging voltage of $30 \mathrm{kV}$. Both the anode and cathode apertures were $3-\mathrm{mm}$ diam. These parameters were chosen from a range of possible parameters and operating frequencies in order to give low jitter in the observed time to breakdown. It is interesting to note that this jitter increases from $50 \mathrm{~ns}$ to more than $10 \mu \mathrm{s}$ when the discharge is changed from operating at $1 \mathrm{~Hz}$ to a single shot basis, suggesting that some long-living species remain in the hollow cathode region for times at least of an order $1 \mathrm{~s}$, thus reducing the statistical time lag.

Fig. 3(a) is a radial streak picture of the discharge taken at a position of $2 \mathrm{~cm}$ from the anode. Fig. 3(b) is taken at the same position except that the camera aperture is reduced by two stops. The schematic shows the time scale of the streak and the radial dimension. The same scale applies to all streak photographs in the following, with $t=0$ corresponding to the start of the discharge current. It is observed that the discharge takes place uniformly over the whole cross section of the discharge tube at breakdown, and that the luminous plasma lasts for the duration of the current. Similar behavior is observed along different axial positions up to a distance of $3 \mathrm{~cm}$ from the cathode.

Fig. 4(a) is a radial streak taken at a point $2 \mathrm{~cm}$ from the cathode under the same condition as Fig. 3(a). Fig. 4(b) is taken at two lower stops. The discharge is observed to start on the axis and expands over the whole volume in a few nanoseconds. A brighter core region where the discharge originates can be seen throughout the discharge.

Totally different behavior is observed in the hollow cathode region. Fig. 5(a) is a radial streak photograph taken within the hollow cathode at $1-\mathrm{mm}$ behind the back surface of the cathode under the same conditions as those in Fig. 3(a). Fig. 5(b) is taken at two stops less, while Fig. 5(c) is taken at two stops more in order to show up any features of lower or higher luminosity. It can be seen that the emitting region begins from a point on axis, with a typical dimension of less than $0.3-\mathrm{mm}$ diam. This point (a)


Fig. 3. Radial streak photographs of the discharge plasma in the main vol ume taken (a) at $2 \mathrm{~cm}$ along the axis from the anode, and (b) at the same location but with the camera lens apertured down by two stops. The dimension and the time scale are shown on the accompanying sketch. The time " 0 " mark corresponds to the start of the discharge current.



Fig. 4. Radial streak photographs of the discharge plasma in the main volume taken (a) at $2 \mathrm{~cm}$ along the axis from the cathode under the same exposure condition as that of Fig. 3(a), and (b) at the same location but with the camera lens apertured down by two stops. The time " 0 " mark corresponds to the start of the discharge current. The scale sizes are the same as those shown in Fig. 3

grows in size to slightly above $3 \mathrm{~mm}$, the size of the cathode aperture, in about $20 \mathrm{~ns}$. For comparison, the luminosity in the main discharge volume is observed to start at this instant within $2 \mathrm{~ns}$, which is the accuracy of the measurement setup. During the main discharge the plasma inside the hollow cathode is observed to be composed of two regions: A bright core which remains bright for the duration of the discharge, and a less bright plasma which expands to fill the whole of the hollow cathode region and decays faster than the core region.

Axial streak photographs with the slit along the axis of the discharge behind the hollow cathode region are shown 
(a)

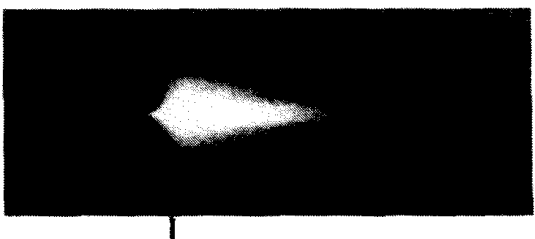

(b)



(c)

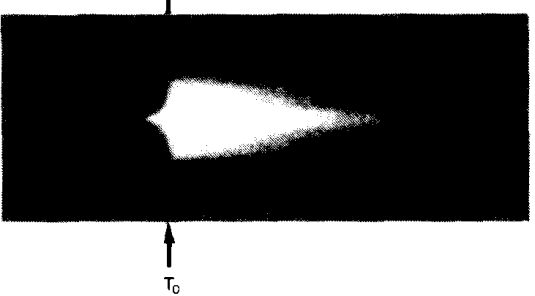

Fig. 5. Radial streak photographs of the discharge plasma inside the hollow cathode at $1 \mathrm{~mm}$ behind the back surface of the cathode (a) taken under the same exposure condition as that of Fig. 3(a). (b) taken with the camera apertured down by two stops, and (c) taken with the camera aperture opened up by two stops. The time " 0 " mark corresponds to the start of the discharge current. The scale sizes are the same as those shown in Fig. 3.

in Fig. 6. Fig. 6(a) is taken at the same exposure level as Fig. 3(a), while Fig. 6(b) is taken at two stops lower. It can be seen that the luminous plasma is initially formed close to the cathode aperture and then extends axially into the hollow cathode region. It is not clear from the present measurements whether the very high expansion velocity observed is due to particle movements or the propagation of a ionization front.

\section{Numerical Simulation}

Numerical modeling based on swarm parameters has been carried out to investigate the plasma formation in the hollow cathode region using a fluid description. The model comprises the time-dependent continuity equations for the ions and electrons, describing their motions as well as the generation of new ones by ionization in the hollow cathode volume, and secondaries at the electrode boundary. Poisson's equation is solved in the whole interior, taking into consideration the presence of space charge. Detailed descriptions of the code and preliminary results have been presented elsewhere [6], [7]. The direct comparison between the numerical and experimental results cannot be made at present because of the different geometry and precise conditions used. However, inside the hollow cathode region the value of the reduced field $E / N$ is of similar magnitude, and the simulation results will thus give a good qualitative description of the main ioni-

(a)



Fig. 6. Axial streak photographs of the discharge plasma inside the hollow cathode along the device's axis (a) taken under the same exposure condition as that of Fig. 3(a). and (b) taken with the camera apertured down by two stops. The back surface of the cathode is at the top marker on the picture. The scale sizes are the same as those shown in Fig. 3.

zation phenomenon. In the following, some salient features of the simulation results will be described. The simulation was carried out in 10 torr $\mathrm{N}_{2}$, with a maximum $E / N$ value of $5 * 10^{-18} \mathrm{Vm}^{2}$ inside the hollow cathode.

When the voltage is applied across the anode cathode gap, a highly divergent field is established inside the hollow cathode. The leakage field is a certain fraction of the applied field, the shape and magnitude of which depends primarily on the size of the cathode hole. Over a long initial buildup phase, when space-charge distortion of the applied field is negligible, the carrier densities increase exponentially. Owing to the curvature of the electric field lines inside the hollow cathode, there is an effective radial focusing of the electrons towards the axis near the aperture. Following the field lines, electrons are extracted continuously through the cathode aperture into the main discharge volume from an early time. This continuous and enhanced loss of electrons leads to the formation of a highly localized positive space charge on axis behind the cathode opening, with the electron density in this region about two orders of magnitude lower than the corresponding ion density.

An ignition phase then follows, during which this positive space charge grows and begins to enhance the applied electric field in the hollow cathode region close to the aperture. The charge carrier densities then grow faster than exponential, with the electron density near the cathode opening increasing by a factor of 100 in about $13 \mathrm{~ns}$, and the ion density by about 10. Finally, in the breakdown phase, the electron density increases at an even greater rate, gaining a factor of 1000 in $2 \mathrm{~ns}$ and reaching a value of $10^{14} \mathrm{~cm}^{-3}$ in an extremely localized point. Fig. 7 shows the electron current density at a point close to the aperture on axis and at $0.5-\mathrm{mm}$ off axis as a function of time, illustrating the highly localized nature of the growth. The simulation is terminated when the high ionization 


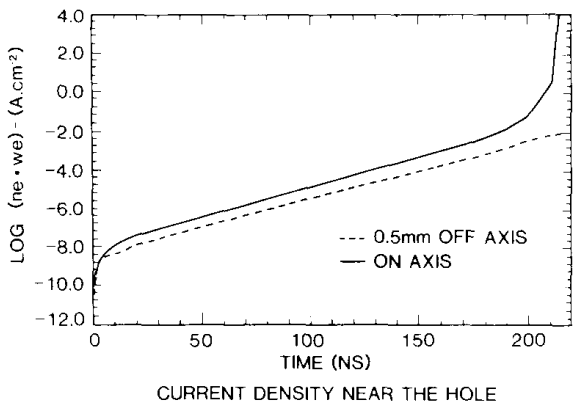

Fig. 7. Numerical simulation results showing the evolution of the electron beam current adjacent to the cathode opening at two radial positions: On axis and at $0.5-\mathrm{mm}$ off axis. The electron beam is directed out through the opening into the main discharge volume.

growth rate invalidates the fluid approach adopted in the code.

\section{Summary and Discussions}

At the low-pressure range and high field of operation in the present experiment, the mean free path of the electron in the main discharge volume is large compared with the tube dimension. Electron collision is thus insufficient to generate the breakdown avalanche, and the breakdown is dependent upon various secondary processes, including secondary electron emission from the cathode. There would be a significant delay in the actual breakdown. For a given pressure, the delay is a function of the number of electrons initially released from the cathode [4].

In a pseudospark discharge, the physics of the discharge initiation is that of a pulsed hollow cathode discharge with a constricted cathode opening. The small aperture in the cathode allows a finite field penetration into the hollow cathode recess. Even though the breakdown process in the main discharge volume is operating on the left-hand side of the minimum in the Paschen curve, inside the hollow cathode the low electric field creates a condition where normal ionization through collision can take place.

In the hollow cathode the applied electric field is divergent, with its highest value on axis immediately behind the aperture. Any electrons created in this region will be extracted through the aperture into the anode/cathode region. The numerical results confirm that the loss of electrons leaves behind a surplus of positive ions, forming a positive space charge. As more electrons drift into this region and are extracted, the field enhancement due to this positive space charge grows and begins to increase the local ionization rate. This results in even more electrons being produced which are then extracted, leaving behind an even higher space-charge field. It is this bootstrap process which rapidly creates a highly localized region of high ionization close to the aperture. This process of plasma formation within the hollow cathode recess agrees with what appears on the streak photographs in Fig. 5 at early times. Finally, the injection of a sufficiently large number of electrons from this source into the main dis- charge volume within a time which is short compared with the transit time initiates the breakdown of the main gap.

The extraction and acceleration of this transient and highly localized source of electrons from behind the cathode into the high field region between the cathode and anode would explain the origin of the high-energy, lowintensity beam measured at low pressures [2]. The explanation, however, could not be extended to the high-current beam observed around the time of the breakdown, as other secondary effects from the main electrodes, and indeed the main discharge volume, must be taken into consideration.

In the main discharge volume the continuous injection of electrons from the hollow cathode region creates an enhanced channel of higher ionization on axis. One would thus expect the discharge current, when breakdown occurs, to flow preferentially down this channel, which in turn would create an even higher degree of ionization. This would explain the concentration of luminosity on axis as observed in the discharge.

The current return path of the present apparatus is in the form of a coaxial return extended from the anode. The electric field is then diverging from the cathode to the anode. Electrons injected from the cathode aperture would move outward, away from the axis, as they drift towards the anode. The differences in the streak photographs near the anode and those near the cathode are consistent with this rather simplified description.

Numerical modeling based on swarm parameters has been carried out to investigate the plasma formation in the hollow cathode region. The results show that electrons are continuously lost through the cathode aperture from an early time in the discharge formation, that there is a creation of a highly localized positive space-charge field on axis behind the cathode recess, and that this space-charge field in turn brings about a super exponential growth of plasma density to $10^{14} \mathrm{~cm}^{-3}$ in a localized region. Although direct comparisons cannot be made with experimental results at present, the simulation demonstrated results that are closely related to the experimental observations. This validates the underlying physics behind the plasma formation within the hollow cathode in a pseudospark discharge. Efforts are being made at present to improve the collision processes included in the model and the associated rate coefficients in order to better model the experiments in the future.

\section{REFERENCES}

11] J. Christiansen and C. Schultheiss, Z. Phisik, vol. A290, p. 35. 1979 [2] P. Choi, H. H. Chuaqui, M. Favre, and E. S. Wyndham. IEEE Trans. Plasma Sci., vol. PS-15, p. 428. 1987

[3] R. B. Quinn, Phis. Rev. vol. 55, p. 482, 1939.

14] J. Lucas, in Proc. Plasma Phys, and Controlled Nacl. Fusion Res. (Vienna. Austria). 1961, p. 693.

[5] D. Bloess et al., Nucl. Instrum. Methods, vol. 205, p. 173, 1983.

[6] K. Mittag and A. J. Davies, in Proc. 18th Int. Conf. on Phenomena in lon. Gases (Swansea. UK), 1987. vol. 4, p. 756

[7] K. Mittag, in Proc. 9th Int. Conf. on Gas Discharges and their Appl. (Venice), 1988, p. 673 


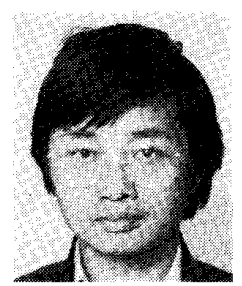

Peter Choi was born in Hong Kong on July 30 1949. He received the B.Sc. degree in physics in 1972 and the Ph.D. degree in 1983 on the studies of pulsed power Z-pinches, both from the Imperial College of Science and Technology, London, England.

From 1977 to the present he has been working in the Plasma Physics group at the Blackett Lab oratory, Imperial College. His research interests include intense plasma $\mathrm{X}$-ray sources, hot spots and electron beams in laboratory plasmas, pulsed power $Z$-pinches, pseudospark discharges, and advanced $X$-ray diagnostics techniques.

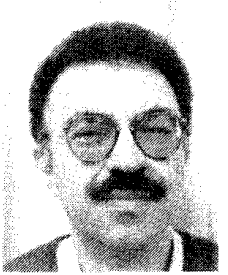

Hernan Chuaqui was born in Santiago. Chile, in 1943. and obtained the electrical engineering degree from the Universidad de Chile in 1969 and the doctorate degree in the studies of confinement in a polytron device from Imperial College. London. England, in 1978

Since 1980 he has been the Head of the Laboratory of Optics and Plasma Physics at the Pontificia Universidad Catolica de Chile, Santiago.
J. Lunney, photograph and biography not available at the time of publication.

R. Reichle, photograph and biography not available at the time of publication.

A. J. Davies, photograph and biography not available at the time of publication. K. Mittag, photograph and biography not available at the time of publi-
cation. 